\title{
Modified economic order quantity (EOQ) model for items with imperfect quality: Game- theoretical approaches
}

\author{
Milad Elyasi, Farid Khoshalhan and Mohammad Khanmirzaee*
}

Department of Industrial Engineering, K.N. Toosi University of Technology, Tehran, IRAN

\section{H R O N I C L E}

\begin{tabular}{l}
\hline Article history: \\
Received April 22013 \\
Received in revised format \\
November 72013 \\
Accepted January 102014 \\
Available online \\
January 262014 \\
\hline Keywords: \\
Inventory management \\
Economic order quantity \\
Game theory \\
Non-cooperative games \\
Cooperative games
\end{tabular}
\begin{abstract}
A B S T R A C T
In the recent decade, studying the economic order quantity (EOQ) models with imperfect quality has appealed to many researchers. Only few papers are published discussing EOQ models with imperfect items in a supply chain. In this paper, a two-echelon decentralized supply chain consisting of a manufacture and a supplier that both face just in time (JIT) inventory problem is considered. It is sought to find the optimal number of the shipments and the quantity of each shipment in a way that minimizes the both manufacturer's and the supplier's cost functions. To the authors' best knowledge, this is the first paper that deals with imperfect items in a decentralized supply chain. Thereby, three different game theoretical solution approaches consisting of two non-cooperative games and a cooperative game are proposed. Comparing the results of three different scenarios with those of the centralized model, the conclusions are drawn to obtain the best approach.
\end{abstract}

\section{Introduction}

Vendor-buyer supply chains have taken on a special significance among the researchers in recent years and the problem of coordination of these supply chains have so far been studied by many researchers. Most of the papers published in this area deal with the supply chain as an integrated system, which leads to the best coordination (see e.g.: Goyal \& Nebebe, 2000; Braglia \& Zavanella, 2003; Wu et al., 2007; Gümüş et al., 2008). Nonetheless, not all of the supply chains are centralized in practice. Accordingly, this magnifies the demand for studying the decentralized supply chains. Centralized models suffer from three major drawbacks. The competitive behavior of the supply chain members despite their cooperative behavior may result in lower efficiency. Furthermore, information gathering for central decision-making would be a very costly action. Finally, if the problem is difficult and large it will be very hard to model and solve the problem (Wang et al., 2004).

The number of the papers discussing decentralized supply chains pursues soaring, as these kinds of problems are similar to real cases. Moses and Seshadri (2000) seek the coordination of a two-echelon

* Corresponding author.

E-mail: M_khanmirzaee@mail.kntu.ac.ir (M. Khanmirzaee)

(C) 2014 Growing Science Ltd. All rights reserved.

doi: $10.5267 /$ j.jijiec.2014.1.003 
supply chain consisting of a manufacturer and a retailer with a make-to-order production policy and propose Nash equilibrium as the solution concept. In order to reach coordination, they consider negotiations on credit terms as cost sharing contracts. Su and Shi (2002) study a supply chain with one manufacturer and one retailer and apply a two-stage game to coordinate the supply chain with a contract with return policy. At first stage, inventory level is determined using Nash equilibrium and in second stage with the help of bargaining, the chain is coordinated. Bylka (2003) applies Nash equilibrium to solve a production-distribution problem in a supply chain. The objective of the model is to minimize the overall costs by finding the number of shipments and the size of production batch per cycle. Introducing a laconic summery of the solution concepts of cooperative and non-cooperative games, Leng and Parlar (2005) present the application of game theory in supply chain in different areas such as inventory games with fix unit purchase cost, inventory games with quantity discount and so on. Gurnani et al. (2007) discuss a supply chain consisting of a supplier and a buyer and study three different scenarios of decision-making structures. Comparing the results of three cases from both the supplier's and the buyer's perspective, they prove that the cost structure and level of uncertainty in demand plays a vital role in players' inventive and is actually major determinant of their choice. Gupta and Weerawat (2006) study a two-layer supply chain with one dominant manufacturer and one supplier and investigate the fact that what mechanisms would the supplier implement to induce the supplier or his/her decision. Those three mechanisms are specifying components inventory level, revenue sharing contract and two-part revenue sharing scheme. Stackelberg equilibrium is used as the solution concept and it is illustrated that the two-part revenue sharing could coordinate the supply chain. A two-echelon supply chain model with one supplier and one manufacturer is developed by Wang et al. (2007). They answer the problem of making a trade-off between vertical integration and outsourcing. Zhou et al. (2008) analyze a supply chain consisted of a manufacturer and a retailer in a fuzzy environment and consider two different situations, firstly, a centralized supply chain is considered, in the second situation, the manufacturer is considered as a leader and subsequently, the retailer as the follower. Hennet and Arda (2008) formulize a model with random demand and random lead-time consisted of a producer and a supplier. The proposed model utilizes queuing theory to evaluate the aspects and game theory for decisional purposes and coordinate the supply chain with application of contracts. Kogan and Tapiero (2010) consider a water supply chain consisting of one supplier and one consumer (buyer) and model the problem as a stochastic game relaxing the assumption that the players are risk-neutral and implement Nash equilibrium to derive the optimal solution. Their study reveals that water-blocking price would have effects on water consumption and consequently the demand for it. Furthermore, they study the situation in which the supply variance depends on the mean supply or not and discuss the result thoroughly. Li et al. (2011) consider supplementary order opportunity in a multi-period inventory problem in a supply chain consisted of a supplier and a buyer and derive optimal production and ordering policies using Nash equilibrium after proving existence and uniqueness of it. Their study illustrates that supplementary supply-order mechanism would result in a better and more efficient supply chain. Chen et al. (2012) discuss a dual-channel supply chain with a manufacturer as a Stackelberg leader and the manufacturer as the follower. Examining coordination schemes, they prove that manufacturer's contract with a wholesale price and the price for the direct channel would coordinate the discussed supply chain, albeit with a benefiting retailer alone. Implementing a complementary agreement, such as two-part tariff or a profit-sharing agreement, they illustrate how the discussed contract may result in a win-win situation. Zhao et al. (2012) propose different scenarios for pricing decisions of a two-stage supply chain with two competitive manufacturer who sale their products by a common retailer. They consider the manufacturing cost and demand with fuzzy uncertainty and implement Nash equilibrium for the game with simultaneous moves and without power imbalance. Wu et al. (2012) consider a supply chain with one supplier and two competing retailers and develop six different scenarios of power imbalance that leads to different sequences of moves among the members of the supply chain. They implement both Nash and Stackelberg equations and compare the gained results. Their analysis reveals that equilibrium values of the models depend on the retail substitutability and when the retail substitutability is low, vertical interaction has more impact than the horizontal interaction. 
A great amount of efforts has been devoted to elaborate and improve basic EOQ model to relax the restricting assumptions of it in order to utilize it in real inventory problems effectively as soon as it was first introduced by Harris (1913). Many papers relaxing the assumption of imperfect items within an inventory problem have been published. Nonetheless, Salameh and Jaber (2000) are the first to study the imperfect items in an EOQ model very thoroughly. They consider an inventory problem in which the demand is deterministic and there is a fraction of imperfect items in the lot and are screened by the buyer and sold by them at the end of the circle at discount price. Nevertheless, there was a problem on calculating the inventory cycle time which was corrected by Cárdenas-Barrón (2000). The research done by Salameh and Jaber (2000) has so far brought up the attention of the researchers on supply chain management in a way that lots of extension of this model have been published. Huang (2002) relaxes the assumption of perfect quality items in supply chain literature. He considers a two-echelon just-in-time (JIT) manufacturing supply chain by accounting for imperfect items. Huang (2004) extends the model introduced by Ha and Kim (1997) and considers the handling of the imperfect items follows the same approach introduced by Salameh and Jaber (2000).

Wahab et al. (2011) consider a vendor-buyer supply chain and study the impact of defected items in three different scenarios consisting of a domestic supply chain, an international supply chain and an international supply chain with considering the environmental impact. A very comprehensive review on extensions of the Salameh and Jaber (2000) EOQ model considering imperfect items has been done by Khan et al. (2011). Introducing the basic model and its assumptions, they study the EOQ models considering imperfect items and the contributions that have been added to the basic model. To the authors' best knowledge, all of the papers published in the literature of imperfect items in supply chains study the supply chain as an integrated system, which is not very adaptive to real life situations. In this paper, we discuss the model which initially introduced by Wahab et al. (2011) with a innovative solution concept by considering the model to be a decentralized one, which is more applicable to real life situations. In doing so, we consider the defected items in a two-echelon decentralized supply chain consisting of a manufacturer and a supplier with three different game theoretical approaches including two non-cooperative and a cooperative approaches as the solution concepts. Nash equilibrium is proposed for the non-cooperative static game and Stackelberg equilibrium is proposed for the noncooperative dynamic game. Furthermore, we propose a cooperative game theoretical approach, which is proved to be Pareto optimal.

The rest of the paper is organized as follows: in section 2, the formulation of the model is described. Section 3 discusses the solution concepts of the problem. Section 4 presents computational results. Section 5 concludes the paper.

\section{Formulation of the model}

The following notation has been used throughout this paper:

$\begin{array}{cl}D & \text { annual demand } \\ x & \text { percentage of the defected items } \\ P & \text { manufacturer's production rate } \\ n & \text { number of shipments determined by the manufacturer } \\ h_{m} & \text { holding cost for the manufacturer per item } \\ A_{m} & \text { preparation cost for the manufacturer per item } \\ F_{m} & \text { fixed transportation cost paid by the manufacturer } \\ C_{m} & \text { variable transportation cost paid by the manufacturer } \\ H_{1_{m}} & \text { holding cost of the producing items for the manufacturer per unit time } \\ H_{2 m} & \text { holding cost of the imperfect items for the manufacturer per unit time } \\ S_{m} & \text { setup cost of the manufacturer per unit time } \\ \operatorname{Tr}_{m} & \text { transportation cost of the manufacturer per unit time }\end{array}$


$T C_{m} \quad$ total cost of the manufacturer per unit time

$Q \quad$ lot size determined by the supplier

$Q_{T} \quad$ total size of the order quantity

$T \quad$ cycle of the supplier

$h_{s} \quad$ holding cost for the supplier per item

$A_{s} \quad$ ordering cost for the supplier per item

$F_{S} \quad$ fixed transportation cost paid by the supplier

$t$ screening time

$y \quad$ screening rate: $y \sim U(0, b)$

$c \quad$ screening cost paid by the supplier

$O_{s} \quad$ ordering cost of the supplier per unit time

$\mathrm{Tr}_{s} \quad$ transportation cost of the supplier per unit time

$H_{S} \quad$ holding cost of the supplier per unit time

$S_{S} \quad$ screening cost of the supplier per unit time

$T C_{S} \quad$ total cost of the supplier per unit time

A two-echelon supply chain consisting of a single manufacturer who sells the products to a single supplier is considered in a single-item-product model in which the manufacturer produces the items with rate of $P$ to meet the constant demand of the supplier for the items $D$. Since no shortage is considered in the proposed model, the production rate must be greater than the demand $(P>D)$. Every time the supplier places an order, the manufacturer transports the demanded items in $n$ equal shipments $\left(Q_{T}=n Q\right)$. Each shipment has $x$ percentage of defected items therefore, the supplier has to scan the received batches for imperfect items. The screen rate of the supplier is $y$ in the time of $t=Q / y$ and the cost of $c$ that is imposed by the manufacturer. The manufacturer has to compensate for the cost of screening of the defected items. When the supplier screens the items, the defected items are stored in their warehouse and the holding cost of the defected items is paid by the manufacturer and at the end of the supplier's inventory cycle when the screening is completed the defect items will be dispatched to the manufacturer and again the manufacturer bears the transportation cost. The total expected cost function of the manufacturer and the one of the supplier will be discussed in this section (Figure 1).

\subsection{Supplier's cost function}

In this subsection, modeling the supplier's cost function is considered. The first step of this modeling is to calculate the cycle of supplier. Every shipment has some defected items, which are not used by supplier. Then the cycle of supplier is obtained by $(1-x) Q / D$. The presence of $\mathrm{x}$ as a random variable in formulation of the cycle, made the supplier's cycle a random variable that its expected value is $(1-E(x)) Q / D$. The total cost of the supplier assumed to be made up by these elements: ordering and transportation cost, holding cost and screening cost.

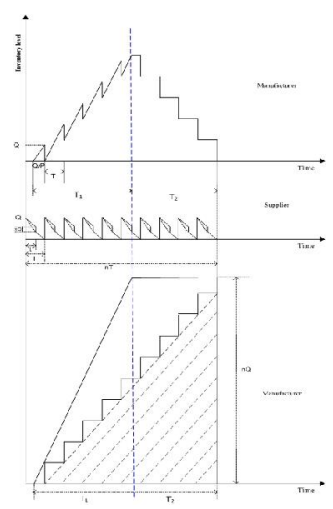

Fig. 1. Total inventory profile for the manufacturer and the supplier. 
a) Ordering and transportation costs:

The fixed ordering cost of $A_{s}$ should be paid by the supplier in every cycle of the manufacturer. Then the supplier's ordering cost in their cycle is obtained by $A_{s} / n$.

The transportation cost for the supplier in every cycle is shown as $F_{S}$. The expected value of the ordering and transportation cost of the supplier per unit time could be expressed as:

$E\left(O_{s}\right)=\left(\frac{A_{s}}{n}\right) / E(T)$

$E\left(\operatorname{Tr}_{S}\right)=F_{S} / E(T)$

\section{b) Holding cost:}

In this paper, it is assumed that the manufacturer pays the holding cost of the imperfect items then the supplier only is responsible for the holding cost of perfect items. Considering related conditions, the expected value of the holding cost of the supplier per unit time can be calculated through following equation:

$E\left(H_{s}\right)=\left(\frac{Q E(1-(x)) E(T))}{2}+\frac{Q^{2} E(x)}{2 y}\right) h_{s} / E(T)$

c) Screening cost: be shown as:

As screening is done for all units in a batch, then the screening cost of supplier per unit time can

$E\left(S_{s}\right)=Q c / E(T)$

In order to obtain the expected total cost of the supplier, the above equations should be added to each other:

$E\left(T C_{s}\right)=E\left(O_{s}\right)+E\left(T r_{s}\right)+E\left(H_{s}\right)+E\left(S_{S}\right)=\frac{F_{s}+\frac{A_{s}}{n}}{E(T)}+\frac{\left(\frac{Q E(1-(x)) E(T))}{2}+\frac{Q^{2} E(x)}{2 y}\right) h_{s}}{E(T)}+\frac{Q c}{E(T)}$

$E(T)$ is replaced with its value, which is $(1-E(x)) Q / D$, then the following equation is obtained:

$E\left(T C_{s}\right)=\frac{D}{(1-E(x))}\left(\frac{1}{Q}\left(\frac{A_{s}}{n}+F_{s}\right)+\frac{Q h_{s}}{2}\left(\frac{E(x)}{y}+\frac{E\left[(1-x)^{2}\right]}{D}\right)+c\right)$

\subsection{Manufacturer's cost function}

The expected total cost of manufacturer per unit time is comprised of holding costs, preparation and transportation cost. The holding cost consists of two parts: holding cost for producing items and for imperfect items, which are in the warehouse of supplier.

\section{a) Holding cost:}

Here, we discuss the parts of the holding costs in more detail. The first part of holding cost, which is related to producing products, is computed as $\frac{[\text { bold area-shaded area }] h_{m}}{n T}$ (Fig. 1): 


$$
E\left(H_{1_{m}}\right)=\left\{\left(n Q\left(\frac{Q}{P}+(n-1) E(T)\right)-\frac{n Q\left(\frac{n Q}{P}\right)}{2}\right)-E(T)(Q+2 Q+\cdots+(n-1) Q)\right\} h_{m} /(n E(T))
$$

The expected value of the holding cost of the imperfect items per unit time, for $n$ cycles of the supplier, which is the second part of the holding costs would be expressed as:

$E\left(H_{2_{m}}\right)=\left(\frac{n Q^{2} E(x)}{2 y}\right) h_{s} / E(T)$

b) Setup and transportation cost:

The setup and preparation cost of $A_{m}$ should be paid by the manufacturer in every cycle of theirs. Because of the existence of defected items in batches, the transportation cost of the manufacturer will be multiplied by $n$. The expected setup and transportation cost of the manufacturer per unit time is represented as:

$E\left(S_{m}\right)=A_{m} /(n E(T))$

$E\left(T r_{m}\right)=n\left(F_{m}+C_{m} E(x) Q /(n E(T))\right.$

Expected total cost of the manufacturer is sum of the above equations. Substituting $E(T)$ by $(1-$ $E(x)) Q / D$, it is rewritten by:

$$
\begin{aligned}
E\left(T C_{m}\right) & =D Q\left(\frac{h_{m}}{P(1-E(x))}\left(1-\frac{n}{2}\right)+\frac{h_{m}}{2 D}(n-1)+\frac{h_{s} E(x)}{2 y(1-E(x))}\right) \\
& +\frac{D}{Q(1-E(x))}\left(F_{m}+\frac{A_{m}}{n}\right)+\frac{C_{m} D E(x)}{(1-E(x))}
\end{aligned}
$$

\section{Solution concept of the problem}

In this section, we discuss the solution concepts, which are used to analyze the situation. Three different solution concepts consist of a non-cooperative static game, a non-cooperative dynamic game and a cooperative game scheme, used in this paper.

\subsection{Non-cooperative static game}

We assumed that the manufacturer and the supplier simultaneously play a non-cooperative game. We used the Nash equilibrium concept to obtain the optimal shipment size and number of shipments. For doing so, the first derivatives of the best responses of the players with respect to $n$ and $Q$, is set equal to zero and by solving this equation set, the Nash equilibrium is calculated as:

$$
\begin{aligned}
Q^{N} & =\frac{\sqrt{z_{1} z_{2}}+y D A_{s}}{\sqrt{2 z_{1}} h_{s}\left[D E(x)+y E\left[(1-x)^{2}\right]\right]} \\
n^{N} & =\frac{\left(\sqrt{z_{1} z_{2}}+y D A_{s}\right)}{2 y D F_{s}}-\frac{A_{s}}{F_{s}} \\
z_{1} & =\frac{D P A_{m}}{h_{m}[(1-E(x)) P-D]} \\
z_{2} & =\frac{D y\left[y h_{m} A_{s}^{2}((1-E(x)) P-D)+4 F_{s} h_{s} P A_{m}\left(D E(x)+y E\left[(1-x)^{2}\right]\right)\right]}{P A_{m}}
\end{aligned}
$$




\subsection{Non-cooperative and dynamic game}

In order to model a non-cooperative dynamic game, the supplier is assumed the leader and the manufacturer as the follower. For analyzing this model, Stackelberg equilibrium is utilized, which long since been implemented by the researchers (see Ertek and Griffin (2002); Qin and Yang (2008); Esmaeili et al. (2009); Chen (2011); Cai et al. (2011); Chen et al. (2012)). Using this approach, the following results are calculated:

$$
\begin{aligned}
& Q^{S}=\sqrt{\frac{2 D F_{s} y}{h_{s}\left(D E(x)+y\left(E[1-x]^{2}\right)\right)}} \\
& n^{S}=\sqrt{\frac{P A_{m} h_{s}\left(D E(x)+y\left(E[1-x]^{2}\right)\right)}{y F_{s} h_{m}((1-E(x)) P-D)}}
\end{aligned}
$$

\subsection{Cooperative game}

According to literature(Peters, 2008), the results of the non-cooperative approaches are not the Paretoefficient. In order to achieve a solution, which is Pareto-efficient, a cooperative game applied. Here we use the cooperative game introduced by Abad and Jaggi (2003). In this game, the weighted sum of the players' objectives is optimized. Assuming that the players agree on the results of the Nash equilibrium:

$$
Z=\lambda\left(T C_{m}\right)+(1-\lambda)\left(T C_{s}\right) \quad 0<\lambda<1
$$

where

$$
\begin{aligned}
& \mathrm{Z}=\lambda\left[D Q\left(\frac{h_{m}}{P(1-E(x))}\left(1-\frac{n}{2}\right)+\frac{h_{m}}{2 D}(n-1)+\frac{h_{s} E(x)}{2 y(1-E(x))}\right)+\frac{D}{Q(1-E(x))}\left(F_{m}+\frac{A_{m}}{n}\right)+\frac{C_{m} D E(x)}{(1-E(x))}\right] \\
& \quad+(1-\lambda)\left[\frac{D}{(1-E(x))}\left(\frac{1}{Q}\left(\frac{A_{s}}{n}+F_{s}\right)+\frac{Q h_{s}}{2}\left(\frac{E(x)}{y}+\frac{E\left[(1-x)^{2}\right]}{D}\right)+c\right)\right]
\end{aligned}
$$

Setting the first order derivative of $Z$ with respect to $n$ equal to zero yields:

$$
\frac{\sigma Z}{\sigma n}=0 \Longrightarrow \lambda=\frac{2 D P A_{m}-h_{m} n^{2} Q^{2}((1-E(x) P-D)}{2 D P\left(A_{m}-A_{s}\right)-h_{m} n^{2} Q^{2}((1-E(x) P-D)}
$$

Now, by setting the first order derivative of $Z$ with respect to $Q$ equal to zero with the given $\lambda$ the optimal size of the shipments would be gained:

$$
Q^{c}=\frac{1}{n h_{m}} \sqrt{\frac{2\left(D h_{m} P\left(A_{m}+A_{s}\right)\right.}{(1-E(x)) P-D}}
$$

\section{Computational results}

\subsection{Numerical example}

To illustrate the efficiency of the model, the numerical example that was introduced by Huang (2004) is implemented and the results are compared with those of the centralized model by Wahab et al. (2011). Let production rate $P=160000$ units/year, demand rate $D=50000$ units/year, holding cost for manufacturer $h_{m}=\$ 2$ unit/year, preparation cost for the manufacturer $A_{m}=\$ 300 /$ cycle, fixed 
transportation cost for manufacturer $F_{m}=\$ 19 /$ delivery, variable transportation cost for manufacturer $C_{m}=\$ 1 /$ unit, ordering cost for the supplier $A_{s}=\$ 100 /$ order, holding cost of the supplier $h_{s}=$ $\$ 5 /$ unit/year, screening cost $c=\$ 0.5 /$ unit, screening rate $y=175200$ unit/year, transportation cost for the supplier $F_{s}=\$ 25 / \mathrm{r}$ delivery, the upper bound of the uniform function of the defected items $b$ is varied between 0.001 and 0.5. By the same token, since there is no guarantee for $n$ to be integer, the algorithm proposed by Wahab et al. (2011) is applied here as well.

Table 1

Results of the non-cooperative static game

\begin{tabular}{lcccccccc}
\hline$b$ & & & & & & \multicolumn{2}{c}{ Centralized } \\
& $n$ & $Q$ & $T C_{S}$ & $T C_{M}$ & $T C$ & $n$ & $Q$ & $T C$ \\
\hline 0.001 & 5 & 953 & 29756 & 7090 & 36846 & 5 & 1056.1 & 36784 \\
0.01 & 5 & 957 & 29873 & 7329 & 37202 & 5 & 1059.4 & 37141 \\
0.02 & 5 & 961 & 30003 & 7597.2 & 37600 & 5 & 1063 & 37540 \\
0.03 & 5 & 965 & 30135 & 7868.1 & 38003 & 5 & 1066.6 & 37944 \\
0.04 & 5 & 970 & 30268 & 8141.8 & 38410 & 5 & 1070.3 & 38352 \\
0.05 & 5 & 974 & 30403 & 8418.3 & 38821 & 5 & 1074 & 38765 \\
0.06 & 5 & 979 & 30539 & 8697.6 & 39237 & 5 & 1077.7 & 39181 \\
0.07 & 5 & 983 & 30677 & 8979.8 & 39656 & 5 & 1081.4 & 39602 \\
0.08 & 5 & 987 & 30816 & 9265 & 40081 & 5 & 1085.2 & 40027 \\
0.09 & 5 & 992 & 30957 & 9553.2 & 40510 & 5 & 1089 & 40457 \\
0.1 & 5 & 996 & 31099 & 9844.4 & 40943 & 5 & 1092 & 40892 \\
0.2 & 5 & 1041 & 32614 & 12934 & 45548 & 5 & 1132 & 45504 \\
0.3 & 5 & 1086 & 34318 & 16386 & 50703 & 5 & 1173.6 & 50660 \\
0.4 & 5 & 1130 & 36248 & 20267 & 56514 & 6 & 1088.7 & 56457 \\
0.5 & 5 & 1173 & 38451 & 24661 & 63112 & 6 & 1132.4 & 63030 \\
\hline
\end{tabular}

\section{a) Non-cooperative static game}

Applying Nash equilibrium model for the decentralized model by utilizing Equations (12), (13), (14) and (15), the results in Table 1 are given, which at the same time compares the results with those of the centralized model for different values of $b$. The more the value of $b$ the more the difference between the total cost of the decentralized supply chain the centralized one. As it was expected from the beginning, the total cost of the decentralized supply chain is higher than that of the centralized one. Nevertheless, the difference between them is actually negligible while the decentralized model is more applicable to real life situations.

Table 2

Results of the non-cooperative dynamic game

\begin{tabular}{lcccccccc}
\hline$b$ & \multicolumn{3}{c}{ Stackelberg Supplier } & \multicolumn{2}{c}{ Centralized } \\
& $n$ & $Q$ & $T C_{S}$ & $T C_{M}$ & $T C$ & $n$ & \multicolumn{2}{c}{$T$} \\
\hline 0.001 & 7 & 707 & 29559 & 7538.1 & 37097 & 5 & 1056.1 & 36784 \\
0.01 & 7 & 710 & 29675 & 7776.6 & 37451 & 5 & 1059.4 & 37141 \\
0.02 & 7 & 713 & 29805 & 8044.2 & 37849 & 5 & 1063 & 37540 \\
0.03 & 7 & 716 & 29937 & 8314.4 & 38251 & 5 & 1066.6 & 37944 \\
0.04 & 7 & 719 & 30070 & 8587.3 & 38657 & 5 & 1070.3 & 38352 \\
0.05 & 7 & 722 & 30204 & 8863 & 39067 & 5 & 1074 & 38765 \\
0.06 & 7 & 726 & 30340 & 9141.6 & 39482 & 5 & 1077.7 & 39181 \\
0.07 & 7 & 729 & 30478 & 9422.9 & 39901 & 5 & 1081.4 & 39602 \\
0.08 & 7 & 732 & 30617 & 9707.2 & 40324 & 5 & 1085.2 & 40027 \\
0.09 & 7 & 735 & 30757 & 9994.4 & 40752 & 5 & 1089 & 40457 \\
0.1 & 7 & 738 & 30899 & 10285 & 41184 & 5 & 1092 & 40892 \\
0.2 & 7 & 771 & 32412 & 13362 & 45774 & 5 & 1132 & 45504 \\
0.3 & 7 & 804 & 34113 & 16796 & 50909 & 5 & 1173.6 & 50660 \\
0.4 & 7 & 840 & 36039 & 20653 & 56692 & 6 & 1088.7 & 56457 \\
0.5 & 7 & 874 & 38237 & 25016 & 63253 & 6 & 1132.4 & 63030 \\
\hline
\end{tabular}


Stackelberg equilibrium is utilized to draw the results of the non-cooperative dynamic game in the decentralized supply chain. Utilizing Eq. (16) and Eq. (17), the optimal number of shipments and the lot size of each shipment are calculated according to Stackelberg equilibrium and results with comparison to the centralized model are given in Table 2. Comparing the results reveals the fact that the total cost of the supply chain with supplier-Stackelberg assumption is higher than the centralized model as well as the results gained by the Nash equilibrium. Nonetheless, the total cost of the supplier is reduced as he acts as the leader and as the value of $b$ gets higher, the difference between the total costs becomes larger.

Table 3

Results of the cooperative game theory

\begin{tabular}{ccccccccc}
\hline$b$ & \multicolumn{3}{c}{ Cooperative } & \multicolumn{2}{c}{ Centralized } \\
& $n$ & $Q$ & $T C_{S}$ & $T C_{M}$ & $T C$ & $n$ & $Q$ & $T C$ \\
\hline 0.001 & 5 & 1079.1 & 29795 & 6991.9 & 36787 & 5 & 1056.1 & 36784 \\
0.01 & 5 & 1082.7 & 29911 & 7232.4 & 37144 & 5 & 1059.4 & 37141 \\
0.02 & 5 & 1086.7 & 30041 & 7502.2 & 37544 & 5 & 1063 & 37540 \\
0.03 & 5 & 1090.7 & 30173 & 7774.7 & 37948 & 5 & 1066.6 & 37944 \\
0.04 & 5 & 1094.8 & 30306 & 8049.8 & 38356 & 5 & 1070.3 & 38352 \\
0.05 & 5 & 1098.9 & 30440 & 8327.8 & 38768 & 5 & 1074 & 38765 \\
0.06 & 5 & 1103.1 & 30576 & 8608.6 & 39185 & 5 & 1077.7 & 39181 \\
0.07 & 5 & 1107.3 & 30714 & 8892.2 & 39606 & 5 & 1081.4 & 39602 \\
0.08 & 5 & 1111.5 & 30853 & 9178.7 & 40031 & 5 & 1085.2 & 40027 \\
0.09 & 5 & 1115.9 & 30993 & 9468.2 & 40461 & 5 & 1089 & 40457 \\
0.1 & 5 & 1120.2 & 31135 & 9760.7 & 40896 & 5 & 1092 & 40892 \\
0.2 & 5 & 1166.9 & 32649 & 12861 & 45510 & 5 & 1132 & 45504 \\
0.3 & 5 & 1220 & 34354 & 16321 & 50675 & 5 & 1173.6 & 50660 \\
0.4 & 5 & 1281 & 36289 & 20206 & 56495 & 6 & 1088.7 & 56457 \\
0.5 & 5 & 1352.2 & 38503 & 24602 & 63105 & 6 & 1132.4 & 63030 \\
\hline
\end{tabular}

\section{c) Cooperative game}

It has been proved that the results obtained from the cooperative game theoretical models are Paretoefficient (Peters 2008). Comparing the results obtained for decentralized model by cooperative game theoretic approach with those of the centralized model, which is known to be the best solution, would be a clear manifestation of this claim. By using equations (19) and (20) and representing the results on Table 3, the straightforward comparison of the results reveals that the cooperative game theoretical models indeed lead to the Pareto-optimal results.

\subsection{Sensitivity analysis}

The sensitivity analysis of the approaches used in this paper is done through assigning different values to $h_{S}$ and $y$. These parameters are focused because of their crucial characteristics in both manufacturer's and the supplier's performances. In order to conclude the effects of these parameters, other parameters are fixed and the value of the $h_{S}$ and $y$ vary. The value of $b$ is set equal to 0.1 . The resultant changes in value of $Q$ related to the different values of $\mathrm{h}_{\mathrm{S}}$ is shown in Figure 2. Moreover, for the cooperative model while calculating the size of the shipments the number of the shipments is considered the same as the static non-cooperative model. Figure 3 shows the changes in value of the same parameters related to changes occur in the value of $y$.

According to the figures, it is concluded that the size of shipment decreases as the value of $h_{S}$ increases. In other words, as the $h_{S}$ increases the value of the $Q$ is decreased which leads to a higher value of $n$. As the value of $y$ is ascended, expectedly, the quantity of each shipment increases and the number of the shipments decreases. However, the effects of its variation on $Q$ and $n$ vanish. 
Consequently, a major reduction in value of $y$ can have the mentioned effects on $Q$ and $n$.
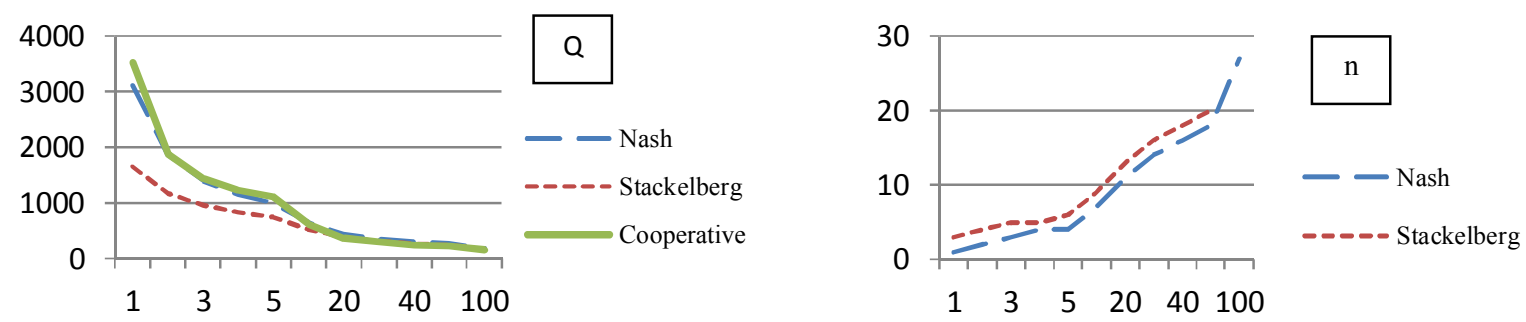

Fig. 2. Effect of $h_{S}$ on $Q^{*}$ and $n^{*}$
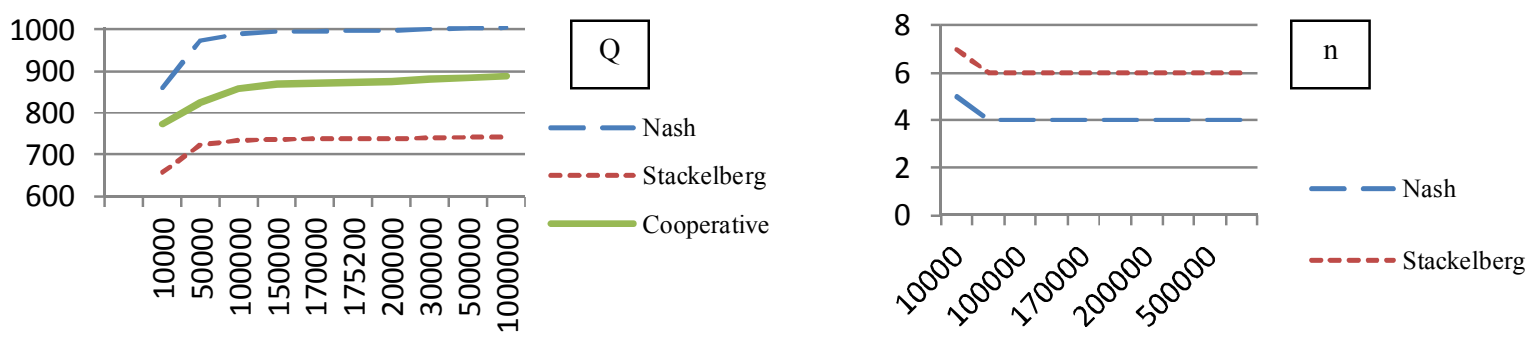

\section{Conclusion}

Fig. 3. Effect of $y$ on $Q^{*}$ and $n^{*}$

A two-echelon supply chain that has been formed of a supplier and a manufacturer was studied in this paper. Here we assume that the imperfect producing is probable, which leads to existence of defected items. The supplier's demand is fulfilled by the manufacturer's products sent in equal sized shipments. Minimizing the total cost of the players by deriving the optimal value of number and the size of shipments is the objective of this paper. In order to analyzed the different relations between players, three different game schemes were used, two non-cooperative and one cooperative model. Nash equilibrium and Stackelberg models with the supplier as the leader are used in non-cooperative models. In cooperative model the weighted sum of total costs of the players minimized leading to a solution, which is Pareto optimal. The effectiveness of the model has been demonstrated by using a numerical example. Furthermore, sensitivity analysis has been accomplished with respect to the parameters, which are vital for players. According to the results obtained through three game models, the result of the cooperative model is the best one and the nearest one to the result of the centralized model. As the centralized model is the best solution, two non-cooperative models do not lead to better result. In Nash equilibrium, the results are near the centralized model but in Stackelberg approach, the supplier as the leader is better off than the other models.

There is a wide scope of future work. For instance:

- The proposed supply chain could be extended to a three-echelon supply chain by adding a downstream customer. In this case, the presence of the imperfect items may lead to back order or lost sale of supplier.

- Relaxation of the assumption that the quantities of shipments are equal could be another relaxation.

- Considering the uncertainty of demand could give results that are more realistic. 
- Considering the asymmetric information model in which the players do not have complete information about each other's cost functions is another scope of future work.

\section{References}

Abad, P. L. \& Jaggi, C. K. (2003). A joint approach for settingunit price and the length of the credit period for a seller when end demand is price sensitive. International Journal of Production Economics, 83, 115-122.

Braglia, M., \& Zavanella, L. (2003). Modelling an industrial strategy for inventory management in supply chains: The 'Consignment Stock' case. International Journal of Production Research, 41(16), 3793-3808.

Bylka, S. (2003). Competitive and cooperative policies for the vendor-buyer. International Journal of Production Economics, 82, 533-544.

Cai, G. G., Chiang, W.-C., \& Chen, X. (2011). Game theoretic pricing and ordering decisions with partial lost sales in two-stage supply chains. International Journal of Production Economics, 130(2), 175-185.

Cárdenas-Barrón, L. E. (2000). Observation on: "Economic production quantity model for items with imperfect quality" [Int. J. Production Economics 64 (2000) 59-64]. International Journal of Production Economics, 67(2), 201.

Chen, J. (2011). The impact of sharing customer returns information in a supply chain with and without a buyback policy. European Journal of Operational Research, 213(3), 478-488.

Chen, J., Zhang, H., \& Sun, Y. (2012). Implementing coordination contracts in a manufacturer Stackelberg dual-channel supply chain. Omega, 40(5), 571-583.

Ertek, G., \& Griffin, P. M. (2002). Supplier- and buyer-driven channels in a two-stage supply chain. IIE Transactions, 34(8), 691-700.

Esmaeili, M., Aryanezhad, M.-B., \& Zeephongsekul, P. (2009). A game theory approach in sellerbuyer supply chain. European Journal of Operational Research, 195(2), 442-448.

Goyal, S. K., \& Nebebe, F. (2000). Determination of economic production-shipment policy for a single-vendor-single-buyer system. European Journal of Operational Research, 121(1), 175-178.

Gümüş, M., Jewkes, E. M., \& Bookbinder, J. H. (2008). Impact of consignment inventory and vendormanaged inventory for a two-party supply chain. International Journal of Production Economics, 113(2), 502-517.

Gupta, D., \& Weerawat, W. (2006). Supplier-manufacturer coordination in capacitated two-stage supply chains. European Journal of Operational Research, 175, 67-89.

Gurnani, H., Erkoc, M., \& Luo, Y. (2007). Impact of product pricing and timing of investment decisions on supply chain co-opetition. European Journal of Operational Research, 180, 228-248.

Ha, D., \& Kim, S.-L. (1997). Implementation of JIT purchasing: An integrated approach. Production Planning \& Control, 8(2), 152-157.

Harris, F. W. (1913). How many parts to make at once. The Magazine of Management, 10(2), 135-136.

Hennet, J.-C., \& Arda, Y. (2008). Supply chain coordination: A game-theory approach. Engineering Applications of Artificial Intelligence, 21(3), 399-405.

Huang, C.-K. (2002). An integrated vendor-buyer cooperative inventory model for items with imperfect quality. Production Planning \& Control, 13(4), 355-361.

Huang, C.-K. (2004). An optimal policy for a single-vendor single-buyer integrated productioninventory problem with process unreliability consideration. International Journal of Production Economics, 91(1), 91-98.

Khan, M., Jaber, M. Y., Guiffrida, A. L., \& Zolfaghari, S. (2011). A review of the extensions of a modified EOQ model for imperfect quality items. International Journal of Production Economics, 132(1), 1-12.

Kogan, K., \& Tapiero, C. S. (2010). Water supply and consumption uncertainty: a conflict-equilibrium. Annals of Operations Research, 181(1), 199-217. 
Leng, M., \& Parlar, M. (2005). Game theoretic applications in supply chain management: a review INFOR, 43(3), 187-220.

Li, X., Li, Y., \& Cai, X. (2011). On a multi-period supply chain system with supplementary order opportunity. European Journal of Operational Research, 209, 273-284.

Moses, M., \& Seshadri, S. (2000). Policy mechanisms for supply chain coordination. IIE Transactions, $32(3), 245-262$.

Peters, H., (2008). Game theory: A Multi-Leveled Approach. Verlag Berlin, Heidelberg: Springer.

Qin, Z., \& Yang, J. (2008). Analysis of a revenue-sharing contract in supply chain management. International Journal of Logistics Research and Applications, 11(1), 17-29.

Salameh, M. K., \& Jaber, M. Y. (2000). Economic production quantity model for items with imperfect quality. International Journal of Production Economics, 64(1-3), 59-64.

Su, C.-T., \& Shi, C.-S. (2002). A Manufacturer's Optimal Quantity Discount Strategy and Return Policy Through Game-Theoretic Approach. The Journal of the Operational Research Society, 53(8), 922-926.

Wahab, M. I. M., Mamun, S. M. H., \& Ongkunaruk, P. (2011). EOQ models for a coordinated twolevel international supply chain considering imperfect items and environmental impact. International Journal of Production Economics, 134(1), 151-158.

Wang, H., Guo, M., \& Efstathiou, J. (2004). A game-theoretical cooperative mechanism design for a two-echelon decentralized supply chain. European Journal of Operational Research, 157(2), 372388.

Wang, L. M., Liu, L. W., \& Wang, Y. J. (2007). Capacity decisions and supply price games under flexibility of backward integration. International Journal of Production Economics, 110(1-2), 8596.

Wu, C.-H., Chen, C.-W., \& Hsieh, C.-C. (2012). Competitive pricing decisions in a two-echelon supply chain with horizontal and vertical competition. International Journal of Production Economics, 135(1), 265-274.

Wu, K.-S., Ouyang, L.-Y., \& Ho, C.-H. (2007). Integrated vendor--buyer inventory system with sublot sampling inspection policy and controllable lead time. International Journal of Systems Science, 38(4), 339-350.

Zhao, J., Tang, W., Zhao, R., \& Wei, J. (2012). Pricing decisions for substitutable products with a common retailer in fuzzy environments. European Journal of Operational Research, 216(2), 409419.

Zhou, C., Zhao, R., \& Tang, W. (2008). Two-echelon supply chain games in a fuzzy environment. Computers \& Industrial Engineering, 55(2), 390-405. 